\title{
Posttraumatic growth enhances social identification in liver transplant patients: a longitudinal study
}

\section{HIGHLIGHTS}

Three main aspects of current literature:

1. A fast growing body of research demonstrates that threatening illnesses such as cancer are often followed by positive life changes and post-traumatic growth (PTG).

1. Establishing and reinforcing interpersonal connections is seen as an important domain of growth in the aftermath of trauma.

2. The bulk of research on the link between trauma and interpersonal relationships is based on cross-sectional data.

Three important extensions of existing literature in the present study:

1. Liver transplant survivors undergo major transformations in terms of one's sense of self, which inevitably impact upon one's interpersonal relationships. The present study is the first one to investigate PTG in liver transplant survivors.

2. This is the first study exploring the impact of PTG on a specific, albeit central dimension of people's life: Social identity. More specifically, this paper looks at the influence of PTG on people's subjective identification with the family group and the group of transplantees.

3. The design of this study is longitudinal. This allows the assessment of the effects of PTG on social identity over time, as well as the possibility of reversed effects. 


\section{Post-traumatic growth enhances social identification in liver transplant patients: a longitudinal study}

Marta Scrignaro, Department of Psychology, University of Milano-Bicocca, Milano, Italy

Fabio Sani, School of Psychology, University of Dundee, Dundee, Scotland, UK

Juliet Ruth Helen Wakefield, Division of Psychology, Nottingham Trent University, England, UK

Elisabetta Bianchi, Clinical Psychology Unit, Fondazione IRCCS Istituto Nazionale dei Tumori, Milano, Italy

Maria Elena Magrin, Department of Psychology, University of Milano-Bicocca, Milano, Italy

Laura Gangeri, Clinical Psychology Unit, Fondazione IRCCS Istituto Nazionale dei Tumori, Milano, Italy

\section{CORRESPONDING AUTHOR}

Dr. Elisabetta Bianchi

Clinical Psychology Unit

Fondazione IRCCS Istituto Nazionale dei Tumori

Via Venezian 1, 20133 Milano, ITALY

Tel: +390223903719; Fax: 02.23902819

Mail: elisabetta.bianchi@istitutotumori.mi.it

WORD COUNT (INTRODUCTION THROUGH DISCUSSION): 2980.

FUNDING: NONE

CONFLICT OF INTEREST: The authors declare no conflict of interest. 


\begin{abstract}
Objective. The main aim of this paper is to investigate the prediction that greater subjective identification with relevant groups and social categories (i.e. 'family' and 'transplantees') can be an outcome of post-traumatic growth (PTG). To date there are no studies that have explored these relationships.
\end{abstract}

Methods. A longitudinal study was conducted with a group of 100 liver transplant patients from the outpatient populations of the participating centre. Data were collected by means of a self-report questionnaire, which was completed at two different time points (T1 and T2) that were 24 months apart. PTG was assessed using the Post-Traumatic Growth Inventory, while both transplantee and family identification were assessed using group identification scales. A path model was tested, using a structural equation model (SEM) approach, to examine the reciprocal effects among family identification, transplantee identification, and PTG over time.

Results. As predicted, we found that greater PTG T1 predicted both greater family identification T2 and marginally greater transplantee identification T2. However, the two identification variables did not predict PTG over time.

Conclusions. The results show that family identification and transplantee identification may be outcomes of the PTG process, confirming the importance of adopting a thriving multidimensional model of adjustment to medical illness, whereby people facing adverse life events, such as transplantation, may flourish rather than deteriorate psychologically.

Keywords: Post-Traumatic Growth; Cancer; Transplant; Social Identification; Social Identity. 


\section{Introduction}

How do people who have survived cancer adjust psychologically to life? Until the mid-1990s, psycho-oncologists assumed that a traumatic life event such as cancer could only cause disruption and distress in one's life. As a consequence, experts considered that one had adjusted to cancer if one showed an absence of diagnosed psychological disorders, no psychological symptoms, no negative mood, or no limitations in physical functioning [1,2]. [2] aptly defined such exclusive focus on negative outcomes as an impairment model of medical illness.

More recently, however, researchers have become aware that positive adjustment is not simply the absence of distress [3], and that many individuals with medical illness such as cancer actually report positive adjustment [4]. As a consequence, an increasing number of researchers have begun to examine the positive outcomes of cancer survivorship [5], thereby proposing a thriving model of illness [2].

One positive outcome of particular interest is post-traumatic growth (PTG) [6-9]. This refers to positive psychological changes in self, life directions, and interpersonal relationships, which are produced by a traumatic life event - for instance a life-threatening illness such as cancer - and may either replace or accompany negative consequences. A rapidly growing literature now shows the high prevalence of positive life changes and personal growth following cancer. Indeed, a recent meta-analysis [10] showed that 60$90 \%$ of people living with cancer report positive changes post-diagnosis.

As mentioned above, researchers consider interpersonal relationships to be an important domain of growth in the aftermath of trauma. Post-trauma interpersonal relationship enhancement is said to take different forms, such as placing increased value on one's relationships with close others, or experiencing greater feelings of compassion and empathy towards those feeling pain or grief [9]. However, according to social psychologists taking a social identity perspective towards human relations $[11,12]$, people do not only establish connections with others as individuals. People may also subjectively identify with (i.e., feel psychologically connected to) social groups and categories, such as one's own family, leisure group, support group, religious community, or people with cancer. Importantly, social identity researchers point to the fact that perceiving others as members of a group with which one identifies is a precondition for experiencing empathy, sympathy, compassion, and concern for others [13, 14]. Put differently, to the extent that one identifies with a group, his or her relationship with other members of that group will be affectively more intense and genuine.

In line with a social identity perspective, the main aim of this paper is to assess the assumption that greater identification with relevant groups and social categories can be an outcome of PTG. Specifically, 
we test the prediction that cancer survivors who have undergone liver transplantation will develop some degree of identification with both their family group and the group of transplantees over time, as a consequence of PTG. This is because people with cancer will normally rely upon family members for moral and instrumental support, and will almost inevitably perceive themselves as members of the category of 'people with cancer'.

Because to date researchers have used group identification exclusively as a predictor of psychological well-being outcomes rather than as a psychological well-being outcome itself $[15,16,17]$, we will also assess the alternative possibility that greater identification with both the family group and the group of transplantees will lead to greater PTG over time.

\section{Method}

\section{Participants and Procedure}

A longitudinal study was conducted from January 2011 to January 2013 at the Istituto di Ricovero e Cura a Carattere Scientifico (IRCCS), the National Cancer Institute of Milan. The study was approved by the ethical committee of the institution in which data were collected.

The participants were liver transplant cancer patients from the outpatient populations of the participating centre. Patients were included in the study if they were 18 years or older, they spoke Italian as their mother tongue, their condition was not so severe that they could not complete the questionnaire, they did not have severe mental disorders or dementia, and they provided written informed consent to participate in the study.

The eligible patients were enrolled in the study by the first author, over a number of visits to the centre. These patients (termed 'participants' from now on) were sent a structured questionnaire asking for various types of medical information, and including various measures of quality of life and PTG. Basic demographic and clinical data were gathered, as well as information on ethnicity and religious affiliation. All questionnaires were self-completed and sent back to the centre inside a pre-stamped envelope. This completed Time 1 (T1) data collection. Two years later, the first author sent the same questionnaire to the same participants, and the procedure was repeated. This completed Time 2 (T2) data collection.

Among the 300 enrolled patients who met the inclusion criteria, 233 (78\%) sent back the questionnaire at T1. Of these 233 individuals, 171 (73\%) sent back the questionnaire at T2. Analyses comparing patients who did not complete the first or the second questionnaire with patients who completed both questionnaires showed no significant differences in socio-demographic characteristics. 
Not all the questionnaires sent back were completely filled in, with 100 (58\%) completely filling in both $\mathrm{T} 1$ and $\mathrm{T} 2$ questionnaires. The statistical analyses presented in this paper concern these 100 participants. It should be noted that this paper reports results from only a portion of these participants' data.

\section{Measures}

\section{Identification With Groups}

Identification with transplantees was assessed with a widely-used four-item global measure of group identification devised by Doosje, Ellemers, and Spears [18]. Items relate to either affective aspects of group identification (e.g., "I feel a link with other people who have had a transplant") or cognitive aspects, specifically self-definitional aspects (e.g., "I see myself as a member of the group of transplantees").

To measure family identification we used the two items related to the affective aspects of group identification from Doosje et al.'s [18] scale; however we replaced the two cognitive items from the scale with two items tapping on perceived similarity with other group members. These items were selected from Leach et al.'s [19] in-group identification scale (e.g., "I have a lot in common with the average member of my family").

Our decision to measure identification in this way was driven by the assumption that the relative importance of the cognitive aspects of group identification may depend on whether the group is a large, relatively abstract social category (such as people who have undergone a transplant), or whether it is a small, intimate group such as the family [20, 21]. In particular, regarding the family group we assumed that one's perceived degree of similarity to other group members is a more relevant manifestation of group identification than self-definition in terms of the group.

In all cases, items were rated on a 7-point scale ( $1=$ I strongly disagree; $7=$ I strongly agree). Each participant's overall transplantee identification was obtained by calculating the mean of their responses to the four transplantee items. Similarly, each participant's overall family identification was obtained by calculating the mean of their responses to the four family items.

\section{Post-Traumatic Growth}

Positive change owing to the liver transplant experience was assessed with the Post-Traumatic Growth Inventory (PTGI) [22]. This is a 21-item measure of growth following an event (e.g., "I changed my priorities about what is important in life"). For each item, participants indicated the degree to which change had occurred in their life 'as a result of having cancer', using a 6-point scale $(0=\mathrm{I}$ did not experience this change; $5=\mathrm{I}$ experienced this change to a very great degree). Although the PTGI consists of five subscales tapping on dimensions such as spiritual change and gain in personal strength, in this study 
we used the inventory as a global measure of PTG because of sample size limitations. This approach, which is consistent with studies that have found the PTGI items to load onto a single factor during exploratory principal component analysis, has been used several times in the literature (e.g., [23, 24]).

\section{Data Analysis}

We began by calculating statistics describing the characteristics of the sample (e.g., age, marital status, etc.) at baseline. At this point we performed two three-factor factor analyses to confirm the legitimacy of treating our three core multi-item variables, namely PTG and the two identification measures, as three independent variables. The first factor analysis concerned the three variables at T1, while the second factor analysis concerned the three variables at $\mathrm{T} 2$. Then we calculated the mean, standard deviation, and reliability of the multi-item variables (i.e., PTG T1 and T2, family identification T1 and T2, transplantee identification T1 and T2), as well as the Pearson's product-moment correlation coefficients for the relationships among these variables and age at baseline. Subsequently we conducted three repeated measures $t$-tests to determine if there were across-time mean differences on PTG, family identification, and transplantee identification. These analyses were conducted using Version 21 of SPSS (Statistical Package for the Social Sciences).

At this point we tested a crossed-lag path model using a structural equation model (SEM) approach. In this case, the analysis was conducted with AMOS 20.0 (Arbuckle, 2011). This model included family identification T1 and T2, transplantee identification T1 and T2, PTG T1 and T2, and age at baseline. This allowed us to test the hypothesised effects of PTG T1 on the identification variables T2, as well as to assess the possibility of reversed effects.

\section{Results}

\section{Preliminary Results}

Baseline characteristics for the sample are presented in Table 1.

The 28 items (21 measuring PTG, 4 measuring transplantee identification, and 4 measuring family identification) were subjected to an exploratory factor analysis (principal axis factoring) in order to investigate whether the items were measuring three separate concepts. The KMO value was .87, and Bartlett's Test of Sphericity was significant, indicating that the assumptions of the analysis were met.

The exploratory factor analysis revealed a five-factor solution (unrotated Eigenvalues $=11.913$, $3.290,2.085,1.367$, and 1.205). Exploring the rotated factors revealed that 11 of the PTG items loaded 
highly onto factor 1 (loadings range $=.377$ to .775 ), while the other 10 items loaded highly onto factor 2 (loadings range $=.427$ to .755 ). Meanwhile, the four transplant identification items loaded highly onto factor 3 (loadings range $=.756$ to .799 ), while the four family identification items loaded highly onto factor 4 (loadings range $=.434$ to .912 ). The pattern for factor 5 was unclear: the highest-loading variable was the first PTG item (.648), while the rest of the variables that loaded onto this factor had loadings below .325 .

However, parallel analysis indicated that only our first three factors yielded significant eigenvalues. We therefore repeated the factor analysis, but explicitly requested 3 factors. Doing this produced a clearer rotated factor structure: the PTG items loaded highly onto the first factor (loadings range $=.460$ to .820 ), while the transplantee identification and family identification items did not load highly (loadings range $=$ .013 to .237 and .050 to .227 respectively). The transplantee identification items loaded highly onto the second factor (loadings range $=.761$ to .806 ), while the PTG and family identification items did not load highly (loadings range $=-.005$ to .441 and .042 to .312 respectively). The family identification items loaded highly onto the third factor (loadings range $=.442$ to .906 ), while the PTG and transplantee identification items did not load highly (loadings range $=.013$ to .324 and .036 to .184 respectively). These results suggest that the PTG items, transplantee identification items, and family identification items measure three separate constructs.

Descriptive statistics for PTG T1 and T2, family identification T1 and T2, and transplantee identification $\mathrm{T} 1$ and $\mathrm{T} 2$, together with reliability analyses, are reported in Table 2. In general, participants had a fairly high level of identification with groups, with scores on transplantee identification T1 and T2 being more variable than scores on family identification T1 and T2. Reliabilities were good for all variables, with Cronbach's alphas ranging from the mid .70s to the mid $.90 \mathrm{~s}$.

\section{Correlational Analyses}

Correlations between the key variables are reported in Table 2. As expected, there were strong positive, statistically significant correlations between T1 and T2 family identification $(r=.67, p<.01)$, T1 and T2 transplantee identification $(r=.69, p<.01)$, and T1 and T2 PTG $(r=.87, p<.01)$. In addition, the two family identification measures had positive, statistically significant correlations with the two transplantee identification measures, with $r$ values ranging from the mid $.20 \mathrm{~s}$ to the low $.40 \mathrm{~s}$. The correlations between PTG and family identification, and between PTG and transplantee identification measures were also positive and statistically significant, with $r$ values ranging from the mid .30 s to the low .40s. Finally, age was negatively correlated with both PTG measures and positively correlated with both family and transplantee identification measures; however these correlations were small and statistically non-significant, except for the correlation between age and T1 family identification $(r=.25, p<.05)$. 


\section{Mean Differences from T1-T2}

Results of repeated measures $t$-tests (with a Bonferroni corrected alpha level of $p<.016$; i.e., .05/3) analyses found no mean differences between T1 and T2 for either PTG; $t(99)=.01, p=1.00$, family identification; $t(99)=1.69, p=.10$, or transplantee identification; $t(99)=-.34, p=.74$

\section{Cross-Lagged Model}

We tested our predictions in a cross-lagged model. This included family identification T1 and T2, transplantee identification T1 and T2, PTG T1 and T2. We excluded age from the model for reasons of parsimony (and because it did not correlate with any other variable). To test the model, we ran a path analysis in AMOS 20.0 (Arbuckle, 2011).

Results are reported in Figure 1. The analysis revealed excellent model fit, as can be expected when all possible paths (except for those between outcome variables) are included, $X^{2}(3)=6.05, p=.11$; CFI $=$ $0.99 ; \mathrm{RMSEA}=0.10 ; 90 \% \mathrm{CI}[0.00,0.22] ; \mathrm{SRMR}=0.02$. All reported regression weights are standardized. As hypothesized, PTG T1 had a positive, statistically significant impact on family identification T2 $($ beta $=.27, p<.001)$. Greater PTG T1 also predicted greater transplantee identification $\mathrm{T} 2$, although the path was only marginally significant $($ beta $=.14, p=.072)$. Neither family identification T1 nor transplantee identification T1 had a statistically significant impact on PTG T2 (beta $=-.06, p=.27$, and beta $=.07, p=.21$, respectively). In addition, family identification T1, transplantee identification $\mathrm{T} 1$ and PTG T1 positively predicted family identification T2 $($ beta $=.63, p<.001)$, transplantee identification $\mathrm{T} 2($ beta $=.56, \mathrm{p}<.001)$ and PTG T2 $($ beta $=.86, p<.001)$ respectively. The $R^{2}$ values for family identification T2, transplantee identification T2, and PTG T2 were .51, .52, and .77 respectively, indicating that the $\mathrm{T} 1$ predictors explained $51 \%, 52 \%$, and $77 \%$ of the variance in the three outcome variables respectively. Overall, this model shows that PTG exerted a positive effect on group identification over time, but not vice-versa. ${ }^{1}$

\footnotetext{
${ }^{1}$ For completeness, we tested the possibility that family identification T2 and/or transplantee identification T2 predicts PTG T2, and, if so, how estimating these additional paths impacts upon the rest of the model. To do this, we added two new paths to the model: from T2 family identification to T2 PTG, and from T2 transplantee identification to T2 PTG. Doing this revealed that the path between T2 family identification and T2 PTG was non-significant (beta $=.05, p=.51$ ), while the path between T2 transplantee identification and T2 PTG was significant (beta $=.15, p=.03)$. We therefore removed the path between T2 family identification and T2 PTG (for reasons of parsimony) and repeated our original path analysis with one additional path between T2 transplantee identification and T2 PTG. Doing this produced an almost identical model to our original, with only three paths (slightly) changing in value: T1 family identification to T2 PTG changed from (beta = -.06, $p=.27)$ to $($ beta $=-.08, p=.12)$, T1 transplantee identification to T2 PTG changed from (beta $=.07, p=.21)$ to $($ beta $=0.02, p=.82)$, and T1 PTG to T2 PTG changed from $(b e t a=.86, p<.001)$ to $($ beta $=.84, p<.001)$. Although fit improved very slightly, $\left(X^{2}(2)=1.31, p=.52 ; \mathrm{CFI}=\right.$ $1.00 ; \mathrm{RMSEA}=0.00 ; 90 \% \mathrm{CI}[0.00,0.18]$; SRMR $=0.01)$, the fact that the Bayesian Information Criterion (BIC) values for the
} 


\section{Discussion}

In line with the social identity perspective [11] and with a thriving model of illness [2], the main aim of this paper was to examine the possibility that cancer survivors who have undergone liver transplantation could develop a stronger identification with both their family group and with the group of transplantees over time, as a consequence of post-traumatic growth. The results of the path analysis broadly confirm this assumption; the process of growth emerged as unique predictor of both transplantee identification and family identification over time.

These results show that, rather than simply being a predictor of health and wellbeing [15], group identification may be an outcome of PTG. This stresses the importance of positive human health [25]. These results also confirm the importance of adopting a thriving multidimensional model of adjustment to medical illness, which may account for the finding that people can psychologically flourish in the face of adverse life events, such as cancer and transplantation [5].

The major strengths of this research are that it is theory driven, uses a longitudinal design, and is the first study to examine the relationship between PTG and group identification. However, this study is not without limitations. First, this study involves a relatively small sample size. This means that interpretation of results must be made with some caution, and that further studies using larger samples need to be conducted in order to confirm our interpretations and assess the extent to which our results may be generalised to larger populations. Another obvious limitation of our study is that only one type of chronic illness, and therefore only one type of traumatic event, was considered. Future research would benefit from the inclusion of patients affected by other chronic illnesses, such as diabetes or multiple sclerosis, or by other traumatic events, such as sexual abuse. Furthermore, our study includes a very diverse sample in terms of the number of years that had passed since the transplants occurred. It is therefore possible that the impact of PTG on group identifications over two years is greater for patients who have undergone the transplant recently than for those who have already lived for many years as transplantees. Unfortunately we could not investigate this issue due to the small size of our sample. However, future research would benefit from comparing a large sample of patients who recently received their transplant with a large sample of patients who received their transplant some time ago. This would enable researchers to investigate whether the relationship between PTG and group identifications is moderated by the length of time since the transplant took place.

two models were almost identical ( 88.944 for the original model and 88.812 for the new model) indicates that there is no real difference between them. Thus, for reasons of parsimony, we retained our original model. 
To conclude, adjusting to life after serious illness may lead to an 'awakening experience', where one comes to realise what really matters [26]. The present study confirms that psychological investment in social groups, or group identification, can be an important outcome of post-traumatic growth in such situations, confirming once again that we are quintessentially and primarily social beings.

\section{Acknowledgments}

We are indebted to the patients who participated in the study. We thank Mrs Paola Serafin, Milda Di Giacomo and Patrizia Rota, from the Gastroenterology, Surgery and Liver Transplantation Unit, Fondazione IRCCS Istituto Nazionale dei Tumori, Milano, Italy, for their valuable help in the collection of clinical data. We also thank Dr. Vincenzo Mazzaferro, from the Gastroenterology, Surgery and Liver Transplantation Unit, Fondazione IRCCS Istituto Nazionale dei Tumori, Milano, Italy, and Dr. Claudia Borreani from the Clinical Psychology Unit, Fondazione IRCCS Istituto Nazionale dei Tumori, Milano, Italy for their precious support in the realization of this study. 


\section{References}

1. Stanton AL, Revenson TA, Tennen H. Health psychology: psychological adjustment to chronic disease. Annu. Rev. Psychol.. 2007 Jan 10;58:565-92.

2. Carver CS. Resilience and thriving: Issues, models, and linkages. J Soc Issues. 1998 Jul 1;54(2):24566.

3. Calhoun LG and Tedeschi RG, editors. Handbook of posttraumatic growth: Research \& practice. Lawrence Erlbaum Associates Publishers, 2006.

4. Mols F, Vingerhoets AJ, Coebergh JW, van de Poll-Franse LV. Quality of life among long-term breast cancer survivors: a systematic review. Euro J Cancer. 2005 Nov 30;41(17):2613-9.

5. Costanzo ES, Ryff CD, Singer BH. Psychosocial adjustment among cancer survivors: findings from a national survey of health and well-being. Health Psychol. 2009 Mar;28(2):147.

6. Joseph S, Wood A. Assessment of positive functioning in clinical psychology: Theoretical and practical issues. Clin Psychol Rev. 2010 Nov 30;30(7):830-8.

7. Linley PA, Joseph S. Meaning in life and posttraumatic growth. J Loss Trauma. 2011 Mar $24 ; 16(2): 150-9$.

8. Joseph S, Linley PA. Positive psychological perspectives on posttraumatic stress: An integrative psychosocial framework. In Joseph S \& Liney PA, editors. Trauma, recovery, and growth: Positive psychological perspectives on posttraumatic stress 2008: 3-20.

9. Tedeschi RG, Calhoun LG. Posttraumatic growth: Conceptual foundations and empirical evidence. Psychol Inq 2004; 15: 1-18.

10. Sawyer A, Ayers S, Field AP. Posttraumatic growth and adjustment among individuals with cancer or HIV/AIDS: A meta-analysis. Clin Psychol Rev. 2010 Jun 30;30(4):436-47.

11. Turner JC, Oakes PJ, Haslam SA, McGarty C. Self and collective: Cognition and social context. Person Soc Psychol Bull 1994; 20: 454-.

12. Haslam SA, van Knippenberg D, Platow MJ, Ellemers N, editors. Social identity at work: Developing theory for organizational practice. Psychology Press, 2014.

13. Reicher S, Spears R, Haslam SA. The social identity approach in social psychology. Sage identities handbook. 2010 Mar 23:45-62.

14. Sani F, Herrera M, Wakefield JRH, Boroch O, Gulyas C. Comparing social contact and group identification as predictors of mental health. Brit J Soc Psychol 2012; 51: 781-90.

15. Jetten J, Haslam C, Haslam SA. The social cure: Identity, health and well-being. Psychology Press, 2012. 
16. Wakefield JR, Bickley S, Sani F. The effects of identification with a support group on the mental health of people with multiple sclerosis. J Psychosom Res. 2013 May 31;74(5):420-6.

17 Sani F, Madhok V, Norbury M, Dugard P, Wakefield JR. Greater number of group identifications is associated with lower odds of being depressed: evidence from a Scottish community sample. Soc Psychiatry Psychiatr Epidemiol. 2015 Sep 1;50(9):1389-97.

18. Doosje B, Ellemers N, Spears R. Perceived intragroup variability as a function of group status and identification. J Exp Soc Psychol 1995; 31: 410-36.

19. Leach CW, van Zomeren M, Zebel S, Vliek ML, Pennekamp SF, Doosje B, Ouwerkerk JW, Spears R. Group-level self-definition and self-investment: a hierarchical (multicomponent) model of in-group identification. J Pers Soc Psychol. 2008 Jul;95(1):144.

20. Forsyth, D. Group dynamics. Cengage Learning, 2009.

21. Ridgeway, CL. The dynamics of small groups. St. Martin's Press New York, 1983.

22. Tedeschi RG, Calhoun LG. The Posttraumatic Growth Inventory: Measuring the positive legacy of trauma. J Trauma Stress. 1996 Jul 1;9(3):455-71.

23. Scrignaro M, Barni S, Magrin ME. The combined contribution of social support and coping strategies in predicting post-traumatic growth: a longitudinal study on cancer patients. Psycho-Oncol. 2011 Aug 1;20(8):823-31.

24. Sheikh AI. Posttraumatic growth in the context of heart disease. J Clin Psychol Med S. 2004 Dec 1;11(4):265-73.

25. Ryff CD, Singer B. The role of purpose in life and personal growth in positive human health. In Wong PTP, Fry PS, editors. The human quest for meaning: A handbook of psychological research and clinical applications. Lawrence Erlbaum Associates, 1998; 213-235.

26. Yalom ID. Staring at the sun: Overcoming the terror of death. The Humanist Psychol. 2008 Nov 18;36(3-4):283-97. 
Table 1. Baseline demographics and medical data presented as number of participants (which is the same as percentages, since $N=100$ ), or as means, standard deviations, and ranges.

\begin{tabular}{llll}
\hline Demographic And Medical Data & Mean (SD) & Range & $n / \%$ \\
\hline
\end{tabular}

Gender

Males

Females

Age in years

Marital Status

Single

Married

Divorced/Separated

Widowed

Not Reported

Education

Primary School

Secondary School (lower level)

Secondary School (higher level)

University Degree
15

85

$59.88(10.09) \quad 25-77$

11

8

3

2

1

21

26

39

14

Type of Treatment

1

2

3
49

49

2 


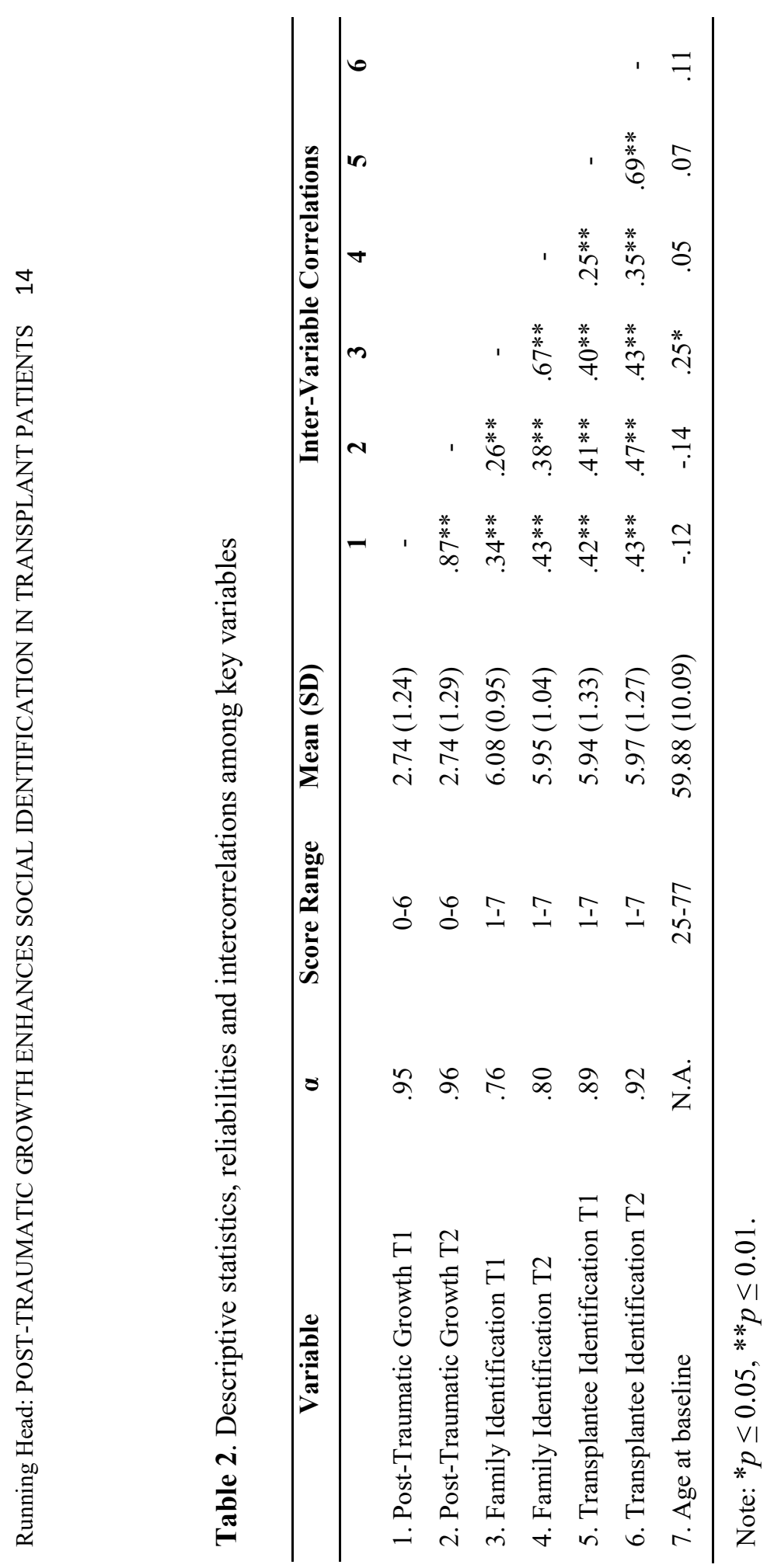




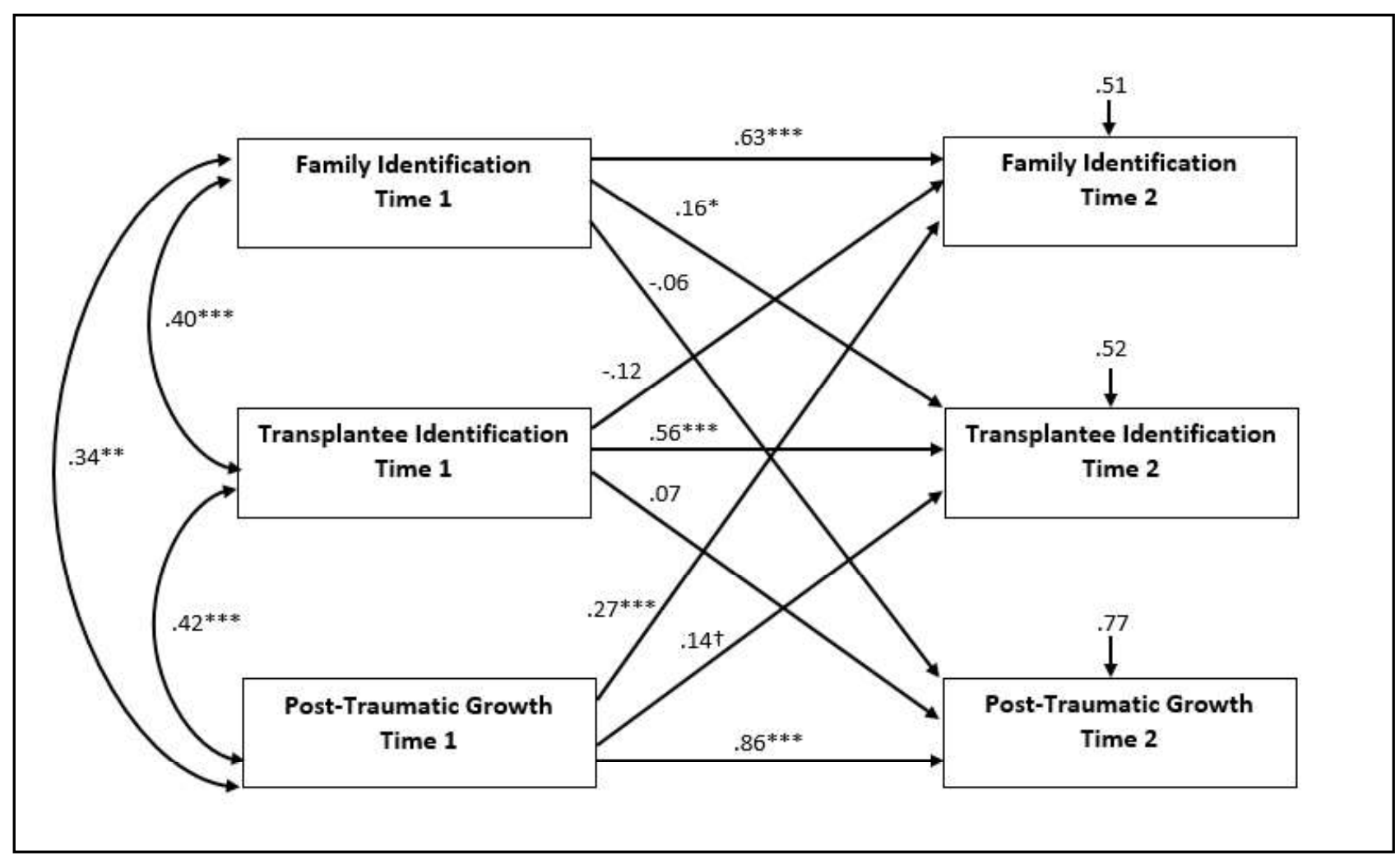

Figure 1. Cross-lagged model testing the relationship between family identification, transplantee identification, and post-traumatic growth (PTG) over time. Note: $\uparrow p=0.072, * p<0.05, * * p<0.01, * * * p<0.001$. 